\title{
A Survey of Cooperative Driving between Auxiliary Autonomous System and Human Driver
}

\author{
Qijie Zou ${ }^{1,2}$, Haoyu Li ${ }^{1,2}$, Rubo Zhang ${ }^{3}$ and Tengda Pei ${ }^{1,2}$ \\ ${ }^{1}$ Information Engineering College, Dalian University, 116622 DaLian, China \\ ${ }^{2}$ BeiDou High Precision Positioning Service Technology Engineering Laboratory, 116622 DaLian, China \\ ${ }^{3}$ Mechatronic Engineering and Automation Institute, Dalian Minzu University, 116600 DaLian, China
}

\begin{abstract}
The cooperative driving is a main direction of intelligent vehicle development since safety-critical tasks must be executed by human and autonomous system. An intelligent vehicle is equipped with a full range of sensors, which are larger and faster than the perception and computational ability of a human driver, while the latter has a comprehensive ability to adapt to unexpected events. According to their respective advantages, the cooperative driving between human and autonomous system can have new synergies. The emphasis of this paper is to survey the current state of the art of cooperative driving, with a specific focus on decision-making and motion planning levels and correlative algorithms. Such researches enable the autonomous system to compensate the human driver in dangerous or uncomfortable circumstance. This paper provides insights into the scope of decision-making and motion planning for cooperative driving, as well as the shortcomings and tendencies.
\end{abstract}

\section{Introduction}

During the past decade, the traffic accidents caused by the improper driving of human driver have increased, and become the main cause of traffic accidents with the increase of the density of vehicles. The improper driving is due to the state of driver, such as tired, distracted, and negligent. Several ambitious projects of autonomous vehicles (or semi-autonomous vehicles), e.g., Google's self-driving car and Tesla's Autopilot, have realized rapid progresses towards commercialization. But from the 2016 Disengagement of Autonomous Mode Report reports [1], self-driving vehicles still need a long time to develop. Meanwhile, intelligent Driver Assistance Systems (DASs) have the potential to improve safety by observing and interacting with the driver. Therefore, there is an increasing interest to keep the human driver in the control loop that needs cooperative driving with the autonomous system to deal with complicated situations. In the field of intelligent vehicle, the study of cooperative control has just started, which is completely different from the existing DASs, since it requires more autonomous driving ability.

The ultimate goal of the cooperative driving system (CDS) focuses on combining the autonomous and the semi-autonomous system with the mature DASs to achieve synergy. Drawing upon fundamental research in understanding of the driver, prediction of vehicle trajectory and the control of collaboration of between human and system, there has been some research progress in this field. To limit the scope of this survey, we focus on the aspects of decision making, motion planning, driver modelling and collaboration controlling for auxiliary autonomous system. When the system finds the deviate trajectory of vehicle, it can predict the driver intention, make decision about the behaviour of vehicle and steer the vehicle safely and smoothly given the driver input.

The remainder of the paper is structure as follows: in section 2, a high level overview of the conceptual architecture and some of key components are presented. Section 3 reviews the decision making and motion planning of autonomous systems, which solve the problem of vehicle trajectory prediction and robust control. Section 4 surveys the understanding of the driver, in order to predict driver input and infer driver intention. The decision methods of collaboration timing are discussed in section 5. Section 6 concludes with remarks on the state of the art and potential areas for future research. Although the intelligent vehicle has no fullautonomous system, we refer to the intelligent control system of vehicle as autonomous system in this paper.

\section{Overview of the framework in CDS}

\subsection{The problem description}

In the CDS, the autonomous driving system collaborates with the driver to steer the vehicle, which can combine 
human's intention with the high-performance computing and full range of sensing capabilities of the intelligent vehicle to achieve the safe, comfort, and low workload driving. Therefore, cooperative driving problem can be described as a synergic effect optimization of two agents, a human driver is natural and an autonomous system is artificial. The graphical representation of this relationship is shown in Figure 1.

The vehicle dynamics is described as $\dot{x}=f(x, u)$. $r_{D}$ and $r_{A}$ are the reference roads of human driver and autonomous system. $u$ is the input into the vehicle dynamics, which is the result of conditional integration of $r_{D}$ and $r_{A}$. If the trajectory prediction is no exception, the driver instruction solely controls the vehicle. Otherwise, the control commands will be based on the vehicle state, the reference path, and the action of the driver. $u=g\left(x, u_{D}, r_{A}\right)$, whereas $u_{D}$ is the input of driver [2-3].



Figure1. The problem description of cooperative driving.

\subsection{General framework}

The main components used in CDS are the hierarchy of decision-making process of autonomous driving system, the cooperative interface and the driver model, cf. Figure 2.

The autonomous driving system is a vehicle selfmanagement agent that generates reference paths and the control command. The cooperative interface controls the integration of human instructions and the reference paths of vehicle when it is aware of unusual driving behaviors. A human driver is the cooperative partner of CDS, which need to understand and predict the intention of human driver.

Therefore, researches in this field depend on the decision-making, motion planning and trajectory prediction of autonomous system, the share control, driver modelling, etc. The subsequent sections review the progresses of related researches.

\subsubsection{Decision making}

As shown in the blue dotted rectangle of figure 1, the hierarchical decision-making process which is a general term for decision-making and planning in autonomous system can be divided into four levels: Route Planning, Behavioral Decision-making, Motion planning, Local Feedback Control. Through the collaboration between the components, system receive real-time environmental information which include the traffic condition of the existing road and driver's own intention to generate global optimal path, obtain the global driving tasks. And then based on some algorithms [4-5], the autonomous system transforms the driving task into the rational driving behavior of the decision, based on the related factors, it is planned to be the driving trajectory which include expected velocity and expected path. Finally, complete the driving.

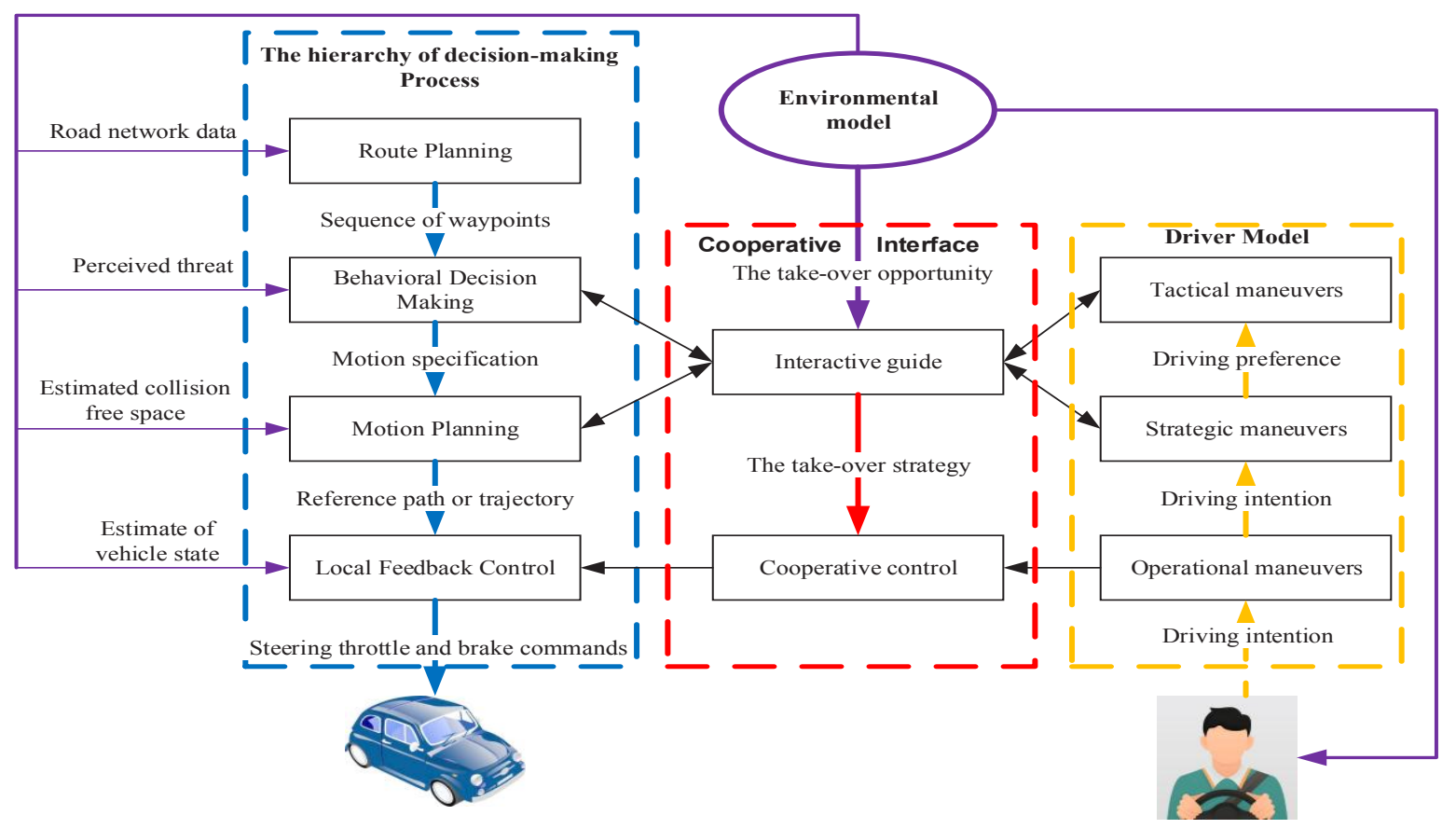

Figure 2. The overall conceptual architecture of cooperative driving. 
Table 1. The introduction of rout planning algorithm.

\begin{tabular}{|c|c|c|c|c|}
\hline Algorithm & Summary & $\begin{array}{l}\text { Development and } \\
\text { application }\end{array}$ & Disadvantage & Solution \\
\hline $\begin{array}{l}\text { Artificial } \\
\text { Potential Field }\end{array}$ & $\begin{array}{l}\text { According to the principle of the } \\
\text { target point, it attracts robot and } \\
\text { obstacles repels robot. It builds a } \\
\text { stepped scalar field and then } \\
\text { finds a feasible path according } \\
\text { to the decline gradient of the } \\
\text { scalar field [12] }\end{array}$ & $\begin{array}{l}\text { Planning aspects of } \\
\text { mobile robots [13-16] }\end{array}$ & \multirow{3}{*}{$\begin{array}{l}\text { The kinematics or } \\
\text { dynamics of } \\
\text { intelligent } \\
\text { vehicles are not } \\
\text { considered in the } \\
\text { planning process, } \\
\text { so that the path of } \\
\text { planning is not } \\
\text { smooth, which is } \\
\text { not conducive to } \\
\text { the following path } \\
\text { tracking. }\end{array}$} & \multirow[t]{2}{*}{$\begin{array}{l}\text { Through geometrical method } \\
\text { to smooth the path planning, } \\
\text { construct basic curve set in } \\
\text { advance, and then use these } \\
\text { basic get planning path curve } \\
\text { are interconnected, making } \\
\text { the whole path is smooth, }\end{array}$} \\
\hline $\begin{array}{l}\text { Methods } \\
\text { Based on } \\
\text { Roadmap }\end{array}$ & $\begin{array}{l}\text { The two visible points are } \\
\text { connected in a straight line to } \\
\text { form a roadmap, and then the } \\
\text { search algorithm is used to find } \\
\text { a feasible path. }\end{array}$ & $\begin{array}{l}\text { Visibility Graph [17- } \\
18] \text { and Voronoi graph } \\
{[19]}\end{array}$ & & \\
\hline $\begin{array}{l}\text { Cell } \\
\text { Decomposition }\end{array}$ & $\begin{array}{l}\text { To divide the whole } \\
\text { environment into convex and } \\
\text { unobstructing regions which are } \\
\text { called units, [20-22] Thus, the } \\
\text { environment is discretized and } \\
\text { the path planning problem is } \\
\text { transformed into a search } \\
\text { problem. }\end{array}$ & $\begin{array}{l}\text { After the map which is } \\
\text { based on the graph } \\
\text { representation is } \\
\text { constructed, the search } \\
\text { algorithm is used to } \\
\text { find the no-collision } \\
\text { path between the } \\
\text { starting point and the } \\
\text { target point. }\end{array}$ & & $\begin{array}{l}\text { By introducing the vehicle's } \\
\text { kinematic and dynamic } \\
\text { constraints to the process of } \\
\text { path planning, with the aid of } \\
\text { vehicle dynamics, to obtain a } \\
\text { more reasonable path } \\
\text { planning, the common } \\
\text { algorithms including the } \\
\text { kinematics model with } \\
\text { curvature polynomial } \\
\text { representation and RRT, etc }\end{array}$ \\
\hline
\end{tabular}

\subsubsection{Cooperative interface}

We summarize the research on the switching and integration of control between drivers and autonomous driving systems, and call it Cooperative Interface. This part includes: Identify the timing of cooperative driving [6-9], and implement a cooperative strategy [10-11]. The former gets the time to identify drivers and autonomous system is identified by means of trajectory prediction. the latter is used to determine who controls or partial control the driver and the autonomous driving system.

\subsubsection{Driver model}

Most autonomous driving systems, whether it's advanced driver assistance systems or the cooperative autonomous driving system that we're concerned about, all they need to be focused on the driver model. In essence, the driver model is the system's description, understanding, evaluation and prediction of the driver, which directly influences the performance of cooperative control between the autonomous driving system and the driver.

Currently, the following research objectives of driver model have been considering by the most scholars and researchers. Firstly, it is the intentional prediction, where the driver model could predict the driving intention of the driver base on its driving prior, and further enhance the cooperation efficiency between the system and driver. The second object is dissociation evaluation, since the model will prevent the improper driving behavior that caused by the driver dissociation. In addition, the evaluation has other functions such as the early warning of driving risk. At last, the driving behavior modelling will perform a analysing of the characteristics of the action of driver's wheel steering, usage of brake and gas pedal, so as to realize personalized driving assistance.

\section{The hierarchy decision making process}

\subsection{Rout planning}

In the CDS, the vehicle is required to have strong autonomous planning and independent decision-making ability to cooperate with the driver at the right time. The rout planning of intelligent vehicle draws on the related research of wheeled robot and develops very rapid.

There are three main types of rout planning methods: artificial potential field [12-16], methods based on roadmap [17-19], and cell decomposition [20-22]. The introduction to these algorithms is shown in Table 1. And also introduce the development and improvement of some of these methods in the field of intelligent vehicle.

In recent years, RRT (rapidly exploring random tree) algorithm and its many variants are also gradually applied in intelligent vehicle path planning [23-25]. As a typical random sampling method, RRT can combine the dynamic constraints of the vehicle during the path planning. However, the sampling efficiency of the algorithm is the main factor affecting the real-time performance of planning, and how to improve the sampling efficiency is a major problem in the future.

\subsection{Behaviour decision making}

Driving behavior decision-making is an important function module of intelligent car, but also the focus and difficulty of autonomous driving technology research. Driving behavior decision-making is based on the perception of the system to obtain environmental information and the current state of the vehicle to make 
safe and reasonable driving operation,such as vehicles to follow, lane, overtak, import traffic, etc., its capability is an important indicator of intelligent vehicle intelligence level indicators.

Rule-based decision-making method is one of the main methods of intelligent vehicle driving behavior decision-making [26], In the 2007 DARPA City Challenge, the Boss Smart Car of CMU (Carnegie Mellon University) used a rule-based approach to automate vehicle driving decisions [27-28]. But the method is robustness for high dynamic traffic environment.

In order to make the driving decision system more robust, Wei et al. [29-30] proposed a method based on PCB (prediction and cost function-based algorithm) to realize the high speed of intelligent vehicle, Naranjo et al. [31] proposed an intelligent vehicle lane changing method based on fuzzy control, and Perez et al. [32] proposed a fuzzy logic-based decision-making system, but fuzzy rules dependent on the relevant empirical knowledge are often difficult to obtain.

There are also some probabilistic methods for driving behavior decisions is proposed [33], such as based on Dynamic Probabilistic Networks (DPN)[34], Bayesian network[35-37], and based on stochastic automates [38]

\subsection{Motion planning}

The movement of the intelligent vehicle can be divided into longitudinal movement and lateral movement [39], and the corresponding control is speed tracking control and path tracking control.
For speed tracking control, commonly used algorithms fall into two categories: control based on dynamic model and control method based on dynamic model. The former includes sliding mode control, PID feedback control based on kinetic model. The latter includes linear PID, fuzzy PID, and adaptive neural network- fuzzy control PID.

\subsubsection{Path tracking control}

Path control, ie, lateral control, common control methods include feedback control based on geometric or kinematic models, PID control, Robust control, synovial control, pole placement, optimal control, model predictive control, The fuzzy control.

The evaluation and description of these algorithms are shown in Table 2. In this table, we introduce the application of various algorithms in speed tracking control and path tracking control. The control methods included in the table are verified by the real vehicle.

The information in the table includes: whether the algorithm needs the vehicle dynamic model or not, Whether the algorithm needs artificial setting parameters or artificial setting rules when applying the algorithm. The accuracy of the proposed algorithm is related to the efficiency of control. And also introduce whether the algorithm apply in the speed planning control or path tracking control and can get the information of the improvement about this algorithm.

We use N to represent NO, Y to YES, U to Unrelated, $\mathrm{R}$ to Related, $\mathrm{P}$ to Path tracking control, and $\mathrm{S}$ to Speed tracking control.

\subsubsection{Speed tracking control}

Table 2. The evaluation and description of motion planning algorithms.

\begin{tabular}{|c|c|c|c|c|c|c|}
\hline Algorithm & $\begin{array}{l}\text { Use the } \\
\text { vehicle } \\
\text { dynamics } \\
\text { model }\end{array}$ & $\begin{array}{l}\text { Need } \\
\text { artificial } \\
\text { parameters } \\
\text { and rules }\end{array}$ & $\begin{array}{l}\text { Relationship } \\
\text { between model } \\
\text { precision and } \\
\text { control effect }\end{array}$ & $\begin{array}{l}\text { Speed } \\
\text { tracking } \\
\text { control }\end{array}$ & $\begin{array}{l}\text { Path } \\
\text { tracking } \\
\text { control }\end{array}$ & Improvement \\
\hline $\begin{array}{l}\text { sliding } \\
\text { mode } \\
\text { control }\end{array}$ & Y & $\mathrm{N}$ & U & $\begin{array}{c}\mathrm{Y} \\
{[40-43]}\end{array}$ & $\begin{array}{c}\mathrm{Y} \\
{[54]}\end{array}$ & 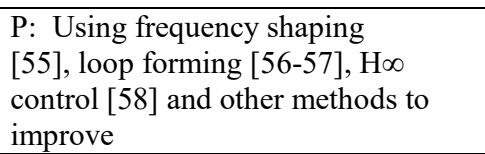 \\
\hline $\begin{array}{l}\text { Feedback } \\
\text { Control } \\
\text { Based on } \\
\text { Dynamic } \\
\text { Model }\end{array}$ & Y & $\mathrm{N}$ & $\mathrm{R}$ & $\begin{array}{c}\mathrm{Y} \\
{[44-47]}\end{array}$ & $\mathrm{N}$ & $\begin{array}{l}\text { S: Consider modeling the whole } \\
\text { dynamic system, such as longitudinal } \\
\text { motion dynamics [44-45] }\end{array}$ \\
\hline $\begin{array}{l}\text { Linear } \\
\text { PID }\end{array}$ & Y & Y & $\mathrm{R}$ & $\begin{array}{c}\mathrm{Y} \\
{[48-52]}\end{array}$ & $\begin{array}{c}\mathrm{Y} \\
{[57]}\end{array}$ & $\begin{array}{l}\text { S: combine with reinforcement } \\
\text { learning algorithm [48] } \\
\text { P: Introduce proportional gain } \\
\text { scheduling [60] and nested PID } \\
\text { [61] to improve the control effect. }\end{array}$ \\
\hline Fuzzy PID & $\mathrm{N}$ & Y & $\mathrm{U}$ & $\begin{array}{c}\mathrm{Y} \\
{[53]}\end{array}$ & Y & $\begin{array}{l}\text { S: Using neural networks to express } \\
\text { fuzzy rules [53] } \\
\text { P: genetic algorithm is introduced in } \\
\text { fuzzy logic to optimize the } \\
\text { membership function and rules } \\
{[62,63]}\end{array}$ \\
\hline
\end{tabular}




\section{Driver Model}

Driver modelling is widely studied and used in the field of intelligent driving. Researchers set up models of the different research priorities with different approaches. Driving tasks are accomplished with the help for collaboration between the driver and the system in CDS, so the mutual perception evaluation and prediction between those two parts are the prerequisite and foundation for ensuring the safety and comfort of driving.[64]

With the help of human-machine interaction technology, the autonomous system can obtain the realtime status information such as: driver's concerns, status, behavior and posture and so on. Based on the information, the system can predict the behavior and intention of the driver and offer an evaluation to the driver. Generally, the work that the driver needs to do in the driving of the vehicle can be divided into two categories: workload and situation awareness, which are the driver factor that most researchers generally agree is used to predict driving efficiency and safety the definition as follows:

1) Situation Awareness: Predict what will happen and react accordingly [65]

2) Workload: Questionnaire or test results which to assess the cost and difficulty of the driver [66]

Based on this, we divide the purpose of driver model into three categories: Prediction of the driver's behaviour, inference of the driver's intention, identification of the driver's cognition.

\subsection{Behavior model}

The driver's behavior modeling which is used to reproduce or predict the behavior of the driver is the basis of the latter two models. Therefore, it can help the autonomous system to better determine the driver's information, and finish the driving work perfectly. The indicators which represent the physical attributes of the driver and reproduces the mathematical expression of the driving process include: steering wheel Angle, speed and other parameters [67]. The behavior of the driver to operate the vehicle, which has strong adaptability, needed to be considered by multiple variables, such as Limb model, the distribution of eye focus area, the use of foot pedals, and so on. We set up a pilot model to analyze and predict driver behavior by HMM (Hidden Markov Model), Bayesian network, machine learning and other methods.[68-69]

\subsection{Inference of driver intention}

After determining the driver's behavior, status and other information, we then model the driver's intention. The same driver's choice of driving behavior with the different intentions in different time may be particularly aggressive or conservative, when dealing with the same driving scenarios, such as overtaking and lane-changing. And the choice of driver behavior in some state, such as drunkenness and fatigue, becomes an unestimable parameter. Therefore, we need to model the driver's intention to solve the problems in these states.

A common driver's intention determination method includes based on driver characteristic and based on vehicles dynamic state. Both of those methods obtain the required data by using vehicle sensor, such as in-vehicle cameras velocity sensor and steering wheel angle sensor, to perception driver intension. But the method based on driver characteristic determine the intension and degree of activity of the driver by using vision sensor to detect relevant characteristics, such as the pupil diameter of the driver. The frequency and time of blinking, hand motion [70-71], head motion [72], foot motion, etc. another method is based on vision sensors, speed sensors, steering wheel angle sensor, and other unit to monitor the vehicle driving speed, traveling path, and leading vehicle distance, and analyses and judge the state of the driver. the measurement reflects the actual driving behavior of the driver, and then deduce the driver's intentions.

\subsection{Cognitive model}

Although a qualified driver's operation to vehicle is within a certain range, and the driver behavior modeling is based on the driver universal model which get rid of the driver's personality. Then with different drivers' physical and psychological differences, there are differences in the number of drivers, such as reaction time, driving skill, age, gender, etc. in other words, different driver have different Driving Style. Based on the previous driver behavior and intention, researchers judge the ability of the driver through a series of subtasks to obtain relevant features, such as reaction time, the driver on the environment target recognition rate [73]. In addition, the current domestic use statistical principles to analyze the correlation between the drivers' response indicators and traffic accidents, and then analyze the differences between different drivers of these indicators. And other country's researchers mainly studied the learning and adaptation process of the driver's adaptive cruise system to analyze driver's dependence on system [74], and evaluation of vehicle track tracking and vehicle driving capability by comparing the actual driving path [75].

In order to establish efficient and accurate driver model, there is no doubt that a great deal of data is necessary to build a model. Thanks to the current sensor and data storage capabilities, the acquisition of large amounts of data has become a breeze. How to use these massive amounts of data is a top priority. In the method to solve the problem of the driver modelling, machine learning method for large data processing has obvious advantages, especially the application of deep learning approach.

\section{The timing of collaboration between driver and autonomous system}

With the help of cooperative driving technology, we dynamically eliminate the cognitive differences between the driver and the autonomous system, achieving 
consultation, learning and enhancing the ability of autonomous systems to deal with uncertainty, diversity and complexity issues. However, it's still a core research how to switch reasonably the right to drive, and efficient to achieve man-machine integration. With the improvement of autonomous system capabilities, this problem will not disappear, but with the enhancing ability to the autonomy of the system, the cooperative driving technology cooperative driving technology will become a part of the field of autonomous interaction.

In Disengagement Report [1], by statistics we can find that due to improper interaction between the two sides caused by the driver involved in improper participation, lack of participation caused by the situation, accounting for nearly half of the total proportion of detachment .Similarly, if the autonomous system find abnormal phenomena, it should predict the result of abnormal driving behavior and summary up the reason, rather than rigidly intervent in the driver control competence to trigger a strong distrust of driver. Therefore, both sides of the CDS in the completion of mutual perception evaluation and prediction under the premise, it need to be further completed, such as the determination of the suitability of the autonomous system, the degree of influence on driving safety after the changed driving right [76], the possibility and cause of manual intervention by the driver on the operation of the autonomous system [77] and so on. And determine the difference between the operation of the driving autonomous system and the independent operation of the driver [78-79] and so on, then determine the timing of the interaction so as to real

\subsection{Based on threat assessment}

It's necessary for cooperative driving technology to determine the time of Man-machine integration. Now the common timing for the determination can be divided into two: (1) Intelligent vehicle system to travel safety and arrive destination for the constraints and objectives; (2) In addition to safety, autonomous intelligent vehicle systems also meet multiple goals, energy consumption, smoothness, stability, etc. The former for this autonomous system cannot complete the driving task of the situation. The latter means that when the performance of the autonomous system is not good for the existing technology, the research work is still focused on the completion of driving tasks. Most of the existing researches belong to the first case.

The threat-based assessment also falls into the first case, it is based on the starting point of the study to predict the collision situation; according to the research perspective, respectively predict the vehicle driving track and other cars road environment in driving track to determine the next period of time (usually $0.5 \sim 5 \mathrm{~s}$ ) the possibility of collision occurred. Meanwhile the car degree of self-confidence is also man-machine rights changing and integration of the main basis [80-81] In other words, if factors that threaten driving safety factors make the system of confidence less than the threshold or the degree of danger is higher than the threshold, the autonomous system will choose to switch to please driver for help or switch the driving right during the driving process

\subsection{Based on trajectory prediction}

Trajectory prediction is also the first case--driving safety requirements. Lane offset as a cause of road accidents is a major reason, is a variety of factors (road conditions, fatigue driving, drunk driving) the combined effect of the results. In general, the autonomous system obtains the road condition environment through the vehicle sensor, based on the prediction of the future state of the vehicle to determine the danger, and then in the planning layer through the visual / auditory / tactile way to pass the dangerous information to the driver, change the driver for dangerous perception and judgment, to assist in driving to avoid or reduce the occurrence of lane departure accidents. Or a system that corrects the vehicle's travel trajectory when the danger is determined, by controlling the steering / braking / driving control and obtaining the driving right when the vehicle is about to be out of the lane.

The common approach to the timing of man-machine integration, which includes threat assessment or trajectory prediction, can still be seen as a solution to the path planning and decision-making problems in the driving process. In these solutions, reinforcement learning method of multi-objective has just need through the interaction with the environment can get optimal or approximate optimal learning strategies, the advantages of application in the future research is worth looking forward to.

\section{Future researches}

From the above survey, the conclusion can be reached that CDS is a fusion technology of different agents, which can achieve synergy and its research will promote the development of driverless vehicle technology.

The motion planning and decision-making researches have been mature, but the understanding and description of drivers are still in the initial stage of research. Furthermore, the dynamic integration of natural intelligence and artificial intelligence is the key challenge problem.

Thus, first extension of the CDS could be learning ability. CDS will learn driving experience through daily driver behaviors, and establish vehicle decision-making and planning model, which can make the system supplement the driver's actions when detecting dangerous and abnormal. Secondly, a new pattern humanoid intelligent vehicle will be reach Level 3 and Level 4 which can switch the control smoothly between the human and the autonomous system.

\section{References}

1. URL:https://www.dmv.ca.gov/portal/dmv/detail/vr/a utonomous/disengagement_report_2016

2. R Li, et al.arXiv preprint arXiv: $1 \overline{704.00866 ~(2017) . ~}$ 
3. V.A Shia, et al. IEEE Transactions on Intelligent Transportation Systems 15(6), 2696-2709(2014).

4. D.C.K Ngai, et al, Intelligent Transportation Systems Conference, (2007).

5. D.C.K Ngai, N.H.C Yung, IEEE Transactions on Intelligent Transportation Systems, 12(2): 509522(2011).

6. M.M Minderhoud. Towards The New Horizon Together. Proceedings Of The 5TH World Congress On Intelligent Transport Systems, Paper NO. 2145, (Korea, 1998).

7. P. Fancher, Research on desirable adaptive cruise control behavior in traffic streams. (UMTRI-200314, 2003)

8. T.P Alkim, G Bootsma, S.P Hoogendoorn. Intelligent Vehicles Symposium, IEEE 1198-1203 (2007)

9. F. Viti, S.P. Hoogendoorn, T.P. Alkim, et al, Intelligent Vehicles Symposium, 745-750 (IEEE,2008)

10. G.A. Mann, N. Small, Human System Interactions (HSI), 2012 5th International Conference on. IEEE, 35-42, (2012)

11. B.D. Argall, T.D. Murphey, Proceedings of the AAAI Fall Symposium on Artificial Intelligence for Human-Robot Interactions (2014)

12. O. Khatib, International Journal of Robotics Research5(1),90-98(1986)

13. J. Barraquand, B. Langlosi, J.C. Latombe, IEEE Transactions on Systems, Man, and Cybernetics 22(2), 224-241(1992)

14. E. Rimon, D.E. Koditschek, IEEE Transactions on Robotics and Automation,8(5), 501-518(1992)

15. J. Chuang, N. Ahuja, IEEE Trans. Syst., Man, Cybern. B, Cybern28(5), 729-736(1998)

16. S.S. Ge, Y.J. Cui, IEEE Transactions on Robotics and Automation16(5), 615-620(2000)

17. T. Lozano-Perez, Communications of the ACM22, 560-570(1979)

18. H. Rohnert, Information Processing Letters23, 7176(1986)

19. O. Takahashi, R.J. Schilling, IEEE Transactions on Robotics and Automation 5(2), 143-150(1989)

20. J.T. Schwartz, M. Sharir, IEEE Trans. Robot. Autom RA-36(3), 345-398(1983)

21. R.A. Brooks, IEEE Transactions on Systems, Man, and Cybernectics SMC-13(3), 190-197(1983)

22. R.A. Brooks, T. Lozano-Perez, IEEE Transactions on Systems, Man, and Cybernetics SMC-15(2), 224233(1985)

23. W. Xu, J. Wei, J.M. Dolan, et al, Robotics and Automation (ICRA), 2012 IEEE International Conference on. IEEE, 2061-2067(2012).

24. L. Ma, J. Xue, K. Kawabata, et al, IEEE Transactions on Intelligent Transportation Systems16(4), 19611976(2015)

25. M. Du, T. Mei, H. Liang, et al, Sensors16(1), 102(2016)

26. A, Niehaus, R.F. Stengel, IEEE Transactions on Vehicular Technology43(3), 626-634(1994)

27. C. Urmson, J. Anhalt, D. Bagnell, et al, Journal of Field Robotics25(8), 425-466(2008)
28. C.R. Baker, J.M. Dolan, International Cofference on Intelligent Robots and Systems, 1752-1758(2008).

29. J. Wei, J.M. Dolan, 2009 IEEE Intelligent Vehicle Symposium, 1015-1020(2009)

30. J. Wei, J.M. Dolan, B. Litkouhi, 2010 IEEE Intelligent Vehicles Symposium, 512-517, (University of California, San Diego, CA, USA, 2010)

31. J.E. Naranjo, C. Gonzalez, R. Garcia, et al, IEEE Transactions on Intelligent Transportation Systems9(3), 438-450(2008)

32. J. Perez, V. Milanes, E. Onieva, et al, Proceedings of the 2011 IEEE International Conference on Mechatronics, 188-193, (Istanbul, Turkey, 2011)

33. M. Ardelt, C. Coester, N. Kaempchen, IEEE Transactions on Intelligent Transportation Systems13(4), 1576-1585(2012)

34. J. Forbes, T. Huang, K. Kanazawa, In Proceedings of the International Joint Conference on Artificial Intelligence, 1878-1885(San Francisco, CA, USA, 1995)

35. R. Schubert, K. Schulze, G. Wanielik, IEEE Transactions on Intelligent Transportation Systems11(3), 607-616(2010)

36. R. Schubert, G. Wanielik, IEEE Intelligent Transportation Systems Magazine, 6-19(2011)

37. R. Schubert, IEEE Transactions on Intelligent Transportation Systems13(1), 354-364(2012)

38. C. Unsal, Intelligent navigation of autonomous vehicles in an automated highway system: Learning methods and interacting vehicles approach, (1997)

39. M. Mitschke, H. Wallentowitz. Dynamik der kraftfahrzeuge, (Berlin, Springer, 1972)

40. S.B. Choi, J.K. Hedrick, Proceedings of the 1995 Vol5, 3106-3110, (American Control Conference, 1995)

41. J.C. Gerdes, J.K. Hedrick, Control Engineering Practice5(11), 1607-1614(1997)

42. R. Rajamani, H.S. Tan, B.K. Law, et al, IEEE Transactions on Control Systems Technology8(4), 695-708(2000)

43. X.Y. Lu, J.K. Hedrick, 2003. Proceedings of the 2003Vol 1, 36-41(American Control Conference, 2003)

44. D. Anderson, Splined speed control using SpAM (Speed-based Acceleration Maps) for an autonomous ground vehicle, (2008)

45. P.N. Currier, Development of an automotive ground vehicle platform for autonomous urban operations, 2008.

46. F.W. Rauskolb, K. Berger, C. Lipski, et al, Journal of Field Robotics25(9), 674-724(2008)

47. J. Bohren, T. Foote, J. Keller, et al, Journal of Field Robotics 25(9), 598-614(2008)

48. I. Miller, M. Campbell, D. Huttenlocher, et al, Journal of Field Robotics25(8), 493-527(2008)

49. S. Moon, I. Moon, K. Yi, Control Engineering Practice17(4), 442-455(2009)

50. S. Thrun, M. Montemerlo, H. Dahlkamp, et al, Journal of field Robotics23(9), 661-692(2006)

51. J. Leonard, J. How, S. Teller, et al, Journal of Field Robotics25(10), 727-774(2008) 
52. B.J. Patz, Y. Papelis, R. Pillat, et al, Journal of Field Robotics25(8), 528-566(2008)

53. H. Khayyam, S. Nahavandi, S. Davis, Expert systems with applications39(3), 3874-3885(2012)

54. C. Hatipoglu, U. Ozguner, K.A. Redmill, IEEE transactions on intelligent transportation systems4(1), 13-22(2003)

55. H.S. Tan, J. Guldner, S. Patwardhan, et a, IEEE/ASME transactions on mechatronics4(3), 258272(1999)

56. J.Y. Wang, M. Tomizuka, Asian Journal of Control2(3), 140-154(2000)

57. M. Tai, P. Hingwe, M. Tomizuka, IEEE/ASME Transactions on Mechatronics9(4), 609-618 (2004)

58. C. Urmson, J. Anhalt, D. Bagnell, et al, Journal of Field Robotics25(8), 425-466(2008)

59. G.V. Raffo, G.K. Gomes, J.E. Normey-Rico, et al, IEEE transactions on intelligent transportation systems10(1), 92-102(2009)

60. A. Broggi, M. Bertozzi, A. Fascioli, et al, International Journal of Intelligent Control and Systems3(4), 409-441(1999)

61. R. Marino, S. Scalzi, M. Netto, Control Engineering Practice19(12), 1459-1467(2011)

62. E. Onieva, J.E. Naranjo, V. Milanés, et al, Applied Soft Computing11(1), 1303-1309(2011)

63. J. Guo, P. Hu, L. Li, et al, IEEE Transactions on Vehicular Technology61(7), 2913-2924(2012)

64. S.A. Mostafa, M.S. Ahmad, A. Ahmad, et al, 2016 2nd International Symposium on. IEEE, 106111(ISAMSR, 2016).

65. E.C. Adam. Fighter cockpits of the future, 12th DASC, 318-323(, 1993)

66. R, Fuller, Accident Analysis \& Prevention37(3), 461-472(2005)

67. J.L. Coyte, B. Li, H. Du, et alNeural Networks (IJCNN), 2014 International Joint Conference on. IEEE, 940-946(2014)

68. N. Kuge, T. Yamamura, O. Shimoyama, et al, $A$ driver behavior recognition method based on a driver model framework (SAE Technical Paper, 2000)
69. V.A. Shia, Y. Gao, R. Vasudevan, et al, IEEE Transactions on Intelligent Transportation Systems15(6), 2696-2709(2014)

70. C. Tran, M.M. Trivedi, IEEE Transactions on Industrial Informatics8(1), 178-187(2012)

71. M.B. Holte, C. Tran, M.M. Trivedi, et al, IEEE Journal of selected topics in signal processing 6(5), 538-552(2012)

72. E. Murphy-Chutorian, M.M. Trivedi, IEEE Transactions on intelligent transportation systems 11(2), 300-311(2010)

73. J.C.F. De. Winter, R. Happee, M.H. Martens, et al, Transportation research part F: traffic psychology and behaviour, 27, 196-217(2014)

74. C.M. Rudin-Brown, H.A. Parker, Transportation Research Part F: Traffic Psychology and Behaviour, 7(2), 59-76(2004)

75. F. Biral, M. Da. Lio, E. Bertolazzi, Intelligent Vehicles Symposium, 2005. Proceedings, 36-41 (2005)

76. C. Mobus, M. Eilers, Digital Human Modeling, 413422(2009)

77. G.S. Aoude, B.D. Luders, K.K.H Lee, et al, Intelligent Transportation Systems (ITSC), 2010 13th International IEEE Conference on. IEEE, 18551862(2010)

78. T.M. Roehr, Y. Shi, Proceedings of the 10th International Symposium on Artificial Intelligence, Robotics and Automation in Space, 507514(Sapporo, Japan. 2010)

79. Jiajia Chen, Research on Decision Making System of Autonomous Vehicle in Urban Environments (University of Science and Technology of China, 2014)

80. D.I.F. Ferguson, D.A. Dolgov, Modifying behavior of autonomous vehicle based on predicted behavior of other vehicles, (U.S. Patent No. 8,457,827. 4 Jun. 2013)

81. L.O. Stenneth, V. Boroditsky, Mode transition for an autonomous vehicle (U.S. Patent No. 9,365,213. 14 Jun. 2016) 